\title{
ORIGINAL
}

\section{¿Es más eficiente el implante de marcapasos permanentes en hospitales de nivel I?}

\author{
E. Moreno-Millán ${ }^{a}$, J.M. García-Torrecillas ${ }^{b}$, J. Villegas-del Ojo ${ }^{a, *}$ y F. Prieto-Valderrey ${ }^{a}$ \\ a Servicio de Medicina Intensiva, Hospital «Santa Bárbara», Puertollano, España \\ b Servicio de Cuidados Críticos y Urgencias, Complejo Hospitalario «Torrecárdenas», Almería, España
}

Recibido el 12 de abril de 2010; aceptado el 6 de julio de 2010

Disponible en Internet el 8 de agosto de 2010

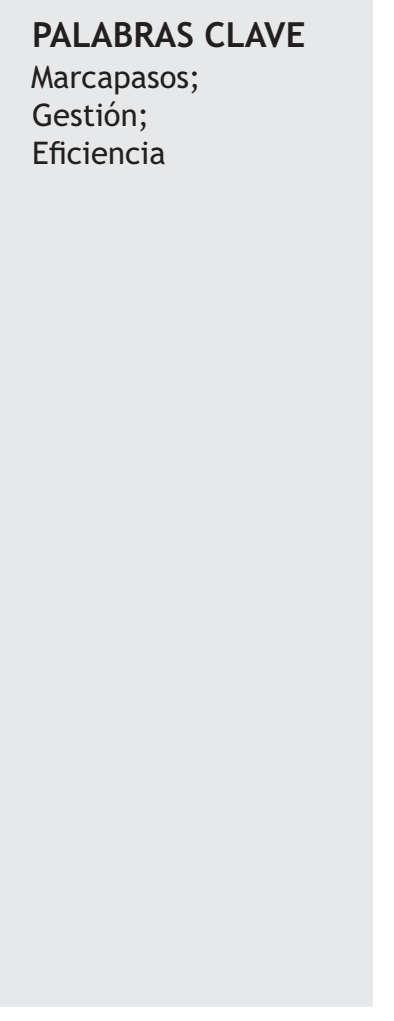

\begin{abstract}
Resumen
Objetivo: Determinar si el implante de marcapasos permanentes (MPP) y cambio de generador resultan más eficientes en hospitales pequeños.

Diseño: Análisis de costeefectividad. Estudio retrospectivo, transversal y observacional de cinco GDR.

Ambito: Los datos son procedentes del conjunto mínimo básico de datos (CMBD) nacional del año 2007, facilitado por el Ministerio de Sanidad.

Pacientes: Son el total de los pacientes que requirieron asistencia en algún hospital nacional por 5 GRD: 115, complicación bradiarrítmica durante la fase aguda de un síndrome coronario, insuficiencia cardíaca o shock; 116, trastorno de conducción sintomático aislado; 117, revisión pero sin cambio de batería; 118, aplicación de una nueva, y 549, implantación o revisión pero con complicaciones graves.

Variables de interés principales: Se analizaron variables demográficas, clínicas (número de diagnósticos secundarios (NDS), de procedimientos (NP), mortalidad) y de gestión (estancia total y preoperatoria (Epo), forma de acceso y alta, tamaño de hospital), definiendo ineficiente una estancia superior 2 días a la media.

Resultados: 23.154 episodios (5,3\% en hospitales $<200$ camas). El estudio bivariado comparativo entre hospitales pequeños y el resto, no discriminado por GDR, mostró estancia media $7,87 \pm 11,01$ días vs $8,78 \pm 12,95$ ( $\mathrm{p}=0,005$, IC $95 \%[0,17 ; 1,65]$ ) y Epo $3,62 \pm 6,14$ vs $4,22 \pm 6,68$ días $[p=0,015])$, sin mayor comorbilidad, medida como proxy por NDS $(5,23 \pm 2,88$ vs $5,42 \pm 3,28$ $[\mathrm{p}=0,055])$; y NP como proxy de esfuerzo diagnóstico-terapéutico $(3,79 \pm 2,50$ vs $3,55 \pm 2,69$ $[\mathrm{p}=0,002]) .24,1 \%$ fueron ineficientes, encontrándose asociación con Epo, NDS, NP y acceso urgente.

Conclusiones: La implantación de marcapasos y cambio de generador en hospitales pequeños es más eficiente, con consistencia interna por subgrupos.

๑ 2010 Elsevier España, S.L. y SEMICYUC. Todos los derechos reservados.
\end{abstract}

\footnotetext{
* Autor para correspondencia.

Correo electrónico: jvdo76@hotmail.com (J. Villegas-del Ojo).
}

0210-5691/\$ - see front matter @ 2010 Elsevier España, S.L. y SEMICYUC. Todos los derechos reservados. doi:10.1016/j.medin.2010.07.002 


\section{KEYWORDS}

Pacemaker; Management; Efficiency

\section{Is the permanent pacemaker implant more efficient in level 1 hospital?}

\begin{abstract}
Objective: To determine if permanent pacemaker implants (PPM) interventions and change of generator are more efficient in small hospitals.

Design: A cost-effective analysis and retrospective, cross-sectional and observational study of diagnostic related groups (DRG).

Setting: The data was obtained from the national Minimum Basic Data Set (MBDS) for the year 2007 provided by the Health Ministry.

Patients: This includes the total number of patients who required treatment in all national hospitals for 5 DRG: 115 - bradyarrhythmic complication during the acute coronary syndrome, heart failure or shock; 116 -symptomatic isolated conduction defects; 117 -revisions, but without changing the battery, 118- application of a new one, 549 - implementation or revision but with serious complications.

Principal variables of interest: demographic, clinical (number of secondary diagnoses (NSD) and procedures (NP), mortality) and management (total and preoperative length of stay (LOS), access, discharge, hospital size), defining inefficient stays as those exceeding 2 days on the average.

Results: 23,154 episodes, $5.3 \%$ small hospitals. The comparative bivariate study between small hospitals and the rest, not discriminated by DRG, showed a mean LOS of $7.87 \pm 8.78$ days vs $11.01 \pm 12.95(\mathrm{p}=0.005,95 \% \mathrm{Cl}$ for mean difference $[0.17,1.65])$ and also lower than preoperatively $(3.62 \pm 6.14$ vs. $4.22 \pm 6.68$ days $(\mathrm{p}=0.015))$ without greater comorbidity, as measured by proxy through the NSD $(5.23 \pm 2.88$ vs $5.42 \pm 3.28(\mathrm{p}=0.055))$ and NP as proxy of diagnostic and therapeutic effort $(3.79 \pm 2.50$ vs $3.55 \pm 2.69(p=0.002))$. A total of $24.1 \%$ were inefficient, there being an association with preoperative stay, NDS, NP and emergency access.

Conclusion: Pacemaker implantation and generator change in small hospitals is more efficient, with internal consistency by subgroups.

(c) 2010 Elsevier España, S.L. y SEMICYUC. All rights reserved.
\end{abstract}

\section{Introducción}

El Catálogo Nacional de Hospitales, creado al amparo de la Ley 16/2003, de Cohesión y Calidad del Sistema Nacional de Salud (SNS), clasifica los centros de la red española según su finalidad asistencial y sus dependencias patrimonial y funcional, respectivamente, pero también contempla cuatro modelos en relación con su capacidad o número de camas y recursos especializados ${ }^{1}$. No obstante, existe una generalizada tendencia a categorizar los hospitales - según su ámbito geodemográfico y su grado de cualificación de oferta - en tres niveles (I, II y III), identificando el primero con centros comarcales de especialidades básicas, habitualmente con menos de 200 camas.

La mayor complejidad de la casuística atendida en los hospitales de nivel superior suele provocar problemas de gestión, dificultando las actividades realizadas alrededor de un mismo proceso asistencial. En este sentido, una de las herramientas empleadas en la medición del producto sanitario es la estancia hospitalaria, generalmente evaluada como variable proxy de su coste directo. Comparar la estancia de unos determinados productos significa, por tanto, comparar el consumo de recursos utilizado en su elaboración. El plan de gestión de una organización es técnicamente eficiente cuando - a partir de unos determinados inputs - genera la máxima cantidad de outputs o se hace en menor tiempo. Una actividad productiva es ineficiente si se pueden reducir la cantidad utilizada de algún input, sin detrimento del resultado, o la duración del proceso.
El primoimplante de marcapasos permanentes (MPP) y la sustitución del generador son dos técnicas habituales en los hospitales, clasificadas administrativamente en cinco grupos de diagnóstico relacionados (GDR): 115, por complicación bradiarrítmica durante la fase aguda de un síndrome coronario, insuficiencia cardíaca o shock; 116, por un trastorno de conducción sintomático aislado; 117 , por revisión pero sin cambio de batería; 118, con aplicación de una nueva, y 549, por implantación o revisión pero con complicaciones graves ${ }^{2}$.

La red hospitalaria pública española está preparada para atender pacientes que precisen la colocación urgente y transitoria de electrocatéter endocavitario con generador externo, pero no todos los hospitales implantan o revisan MPP. Aunque en España existen distintas bases de datos que controlan estas intervenciones (Registros de Marcapasos de la Sociedad Española de Cardiología (SEC) y de la Sociedad Española de Medicina Intensiva, Crítica y Unidades Coronarias (SEMICYUC), respectivamente), muestran diferente número de implantes $\mathrm{y}$, por consiguiente, tasas por millón de habitantes ${ }^{3-5}$. La Base Nacional de Datos de Marcapasos (BNDM) comenzó a funcionar en $1990^{6}$, pero ha sido desde 1993 cuando se han obtenido parámetros comparativos gracias a la introducción de la «tarjeta europea del paciente portador», de obligada cumplimentación ${ }^{7,8}$. Siguiendo, en todo caso, las recomendaciones de la Sociedad Europea de Cardiología9, existen diferencias entre hospitales en España, dependiendo de su tamaño y nivel de oferta, observándose variabilidad en su gestión, según los Registros mencionados ${ }^{3-6}$. 
En muchas ocasiones nos hemos preguntado por qué no se implantan y revisan MPP en todos los hospitales españoles -independientemente de su nivel-contando, por supuesto, con profesionales conocedores de la técnica y con la infraestructura necesaria para ofrecer un producto seguro y de calidad. Y nos hemos planteado si el traslado a otro centro para su colocación supone o no riesgos y retrasos innecesarios para el paciente, molestias a los familiares e incremento de costes, fundamentalmente basados en la prolongación de la estancia, el transporte interhospitalario y los derivados del desplazamiento de acompañantes.

Nuestro objetivo ha sido doble: por una parte, intentar dar respuesta a estas cuestiones como elemento de reflexión para clínicos, gestores y decisores sanitarios y, por otra, analizar el comportamiento de los primoimplantes de MPP y sus revisiones, en relación con el tipo de hospital - clasificado en dos grupos ( $\mathrm{H} 1$ : $<200$ camas, y HR: resto de hospitales)buscando las posibles diferencias de algunos indicadores de gestión (estancia media (Em), preoperatoria (Epo) y necesidad de traslado) y clínicos (complicaciones, comorbilidad y mortalidad), según factores demográficos (edad y género) de los pacientes.

\section{Metodología}

La información procedió del Conjunto Mínimo Básico de Datos (CMBD) del Ministerio de Sanidad y Política Social para el año 2007, facilitada por el Instituto de Información Sanitaria ${ }^{10}$, seleccionándose los casos clasificados como GDR 115 a 118 y 549, prescindiendo de los 849 a 851 (implantes de desfibriladores y resincronizadores). La codificación de los diagnósticos y procedimientos se realizó mediante la Clasificación Internacional de Enfermedades, 9. a edición (MC), y para las agrupaciones de las altas fue utilizado el AP-GDR en su versión 21. A partir de esta información se diseñó un estudio observacional, transversal y retrospectivo con componente inferencial.

Las variables estudiadas fueron la edad (expresada en años y medida al inicio del ingreso), Em y Epo, género, tipo de ingreso (urgente o programado), tipo de alta (domicilio, éxitus, traslado), número y tipología de diagnósticos secundarios al alta (NDS), número de procedimientos efectuados (NP), eficiencia del ingreso y nivel hospitalario. Se consideró ineficiente una estancia superior en dos días a la media, para el GDR en que se encontraba clasificado, ya que seleccionando los casos extremos mediante la fórmula $\mathrm{T} 2=\mathrm{Q} 3+1,5^{*}(\mathrm{Q} 3-\mathrm{Q} 1)$, donde $\mathrm{Q}$ son los cuartiles y $\mathrm{T} 2$ la estancia corte para esos casos, la estancia máxima a considerar era de 22 días, y el análisis de la muestra no outliers revelaba que los percentiles 20,25 y 30 se encontraban con estancias inferiores a dos días, motivo por el que las superiores a este valor se consideraron ineficientes, tras consenso entre los autores sobre el valor de corte.

En una primera fase se procedió al análisis descriptivo de las variables contenidas en el CMBD, utilizando las medidas de posición y dispersión habituales (media, moda y mediana), con sus respectivas desviaciones típicas, para las cuantitativas, y frecuencias, porcentajes y tablas de distribución para las cualitativas. Se aplicó el test de Shapiro-Wilks para determinar el comportamiento en cuanto a normalidad de las variables. En la segunda fase del estudio (bivariante), los contrastes entre las cuantitativas fueron realizados mediante el test de Student para datos independientes. Las comparaciones entre variables nominales, distribuidas en más de dos categorías, se realizaron mediante ANOVA con la prueba post-hoc de máxima diferencia significativa de Tuckey; para las variables categóricas se realizaron contrastes de $\chi^{2}$ no precisándose correcciones por continuidad. Finalmente, para la valoración de la asociación independiente entre estancia eficiente y las diferentes covariables estudiadas, se construyó un modelo de regresión logística binaria con comprobación de la bondad del ajuste mediante el test de Hosmer-Lemeshow, interpretándose los resultados como razones de ventaja (OR) con sus respectivos intervalos de confianza (IC). Y se introdujeron, como variables independientes, las que dieron significativas en el bivariado así como las que -según la bibliografía- consideramos que pudieran estar asociadas a la dependiente. Se tomó como umbral de significación una $\mathrm{p}<0,05$ y fue utilizado el paquete estadístico SPSS 15.0.

\section{Resultados}

Fueron estudiados 23.154 episodios (1\% de los 2.232 .568 totales de personas mayores de 45 años), cuya distribución en función del grupo de hospital se detalla en la figura 1, en la que se observa que solo el $5,3 \%$ de los MPP se implanta en $\mathrm{H} 1$. En la tabla 1 se reflejan los indicadores clínicos y de gestión analizados (Em, Epo, NDS, NP, tipo de ingreso y de alta) así como algunas de las variables demográficas (edad media, porcentaje de pacientes mayores de 70 años, género), según el GDR asignado y el global de los correspondientes a esta línea de producción de MPP. Debe destacarse la superior incidencia $(62,33 \%)$ del GDR 116 , relacionado con trastornos de conducción puros y sin complicaciones, que se producen en pacientes más añosos, con predominio del género masculino e ingreso urgente. El GDR 549 (15,38\% del total) se presenta fundamentalmente en varones $(77,5 \%)$, con Em y Epo muy prolongadas $(24,51$ y 7,63 días, respectivamente) y altas comorbilidad (8,95 diagnósticos secundarios),

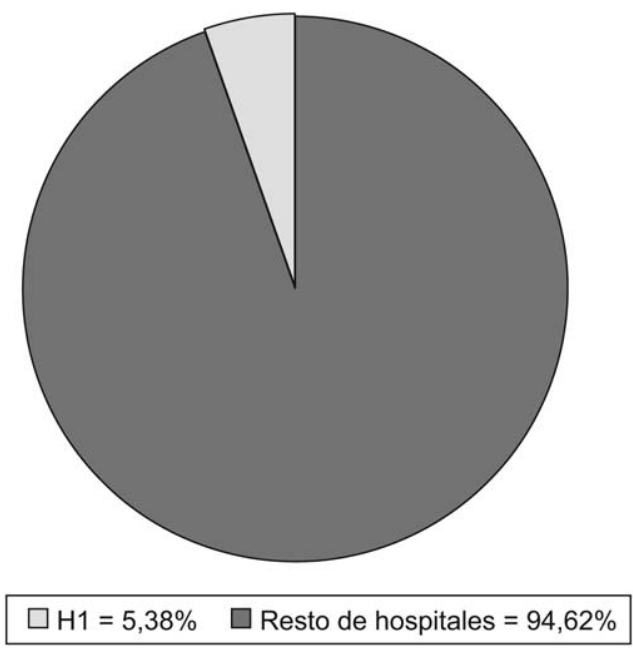

Figura 1 Distribución de los episodios de implantación de marcapasos permanentes según el tamaño de hospital. 
Tabla 1 Indicadores clínicos, demográficos y gestores de los GDR relacionados con marcapasos, España 2007

\begin{tabular}{lllllll}
\hline & Total & 115 & 116 & 117 & 118 & 549 \\
\hline Episodios (N ${ }^{\text {y } \%)}$ & $23.154(100)$ & $813(3,5)$ & $14432(62,33)$ & $1068(4,61)$ & $3280(14,16)$ & $3561(15,38)$ \\
Edad (x DT) & $75,55(9,64)$ & $72,21(10,13)$ & $76,66(8,14)$ & $75,26(9,44)$ & $77,31(9,51)$ & $70,29(10,42)$ \\
$>70$ años (\%) & & 61,8 & 79,0 & 73,2 & 80,6 & 55,3 \\
Varones (\%) & 60,8 & 68,9 & 57,6 & 59,3 & 55,6 & 77,5 \\
Estancia (x DT) & $8,74(12,85)$ & $9,77(9,74)$ & $6,26(5,81)$ & $5,62(6,34)$ & $3,26(3,68)$ & $24,51(24,53)$ \\
E. Preop. (x DT) & $4,17(6,64)$ & $5,25(7,04)$ & $3,77(4,57)$ & $2,65(4,31)$ & $1,58(2,28)$ & $7,63(11,49)$ \\
Urgentes (\%) & 59,5 & 55,6 & 66,8 & 47,6 & 19,7 & 70,8 \\
H1 (\%) & 5,3 & 3,3 & 5,7 & 8,7 & 3,9 & 4,3 \\
NDS (x DT) & $5,42(3,28)$ & $6,39(3,27)$ & $4,99(2,82)$ & $4,70(3,11)$ & $3,42(2,50)$ & $8,94(3,03)$ \\
NP (x DT) & $3,55(2,69)$ & $3,52(2,70)$ & $3,49(1,89)$ & $2,09(1,73)$ & $1,66(1,25)$ & $6,03(4,26)$ \\
Éxitus (por mil) & 45,0 & 15,0 & 4,0 & 18,0 & 5,0 & 268,0 \\
Traslados (por mil) & 14,0 & 10,0 & 9,0 & 18,0 & 5,0 & 42,0 \\
Peso relativo & & 4,6014 & 3,6694 & 2,1114 & 2,0597 & 6,9436 \\
\hline
\end{tabular}

H1 = hospital nivel I; NDS = número de diagnósticos secundarios; NP=número de procedimientos; Instituto de Información Sanitaria, Ministerio de Sanidad y Política Social, 2007.

$(\mathrm{x} \pm \mathrm{DT})=$ media $\mathrm{y}$ desviación típica.

intervenciones diagnóstico-terapéuticas $(6,03)$ y mortalidad (268,0 por mil).

En el estudio bivariado comparativo entre $\mathrm{H} 1$ frente al resto de hospitales de mayor nivel (HR), no discriminado por tipo de GDR, se comprobó que la implantación de un MPP se hacía en $7,87 \pm 11,01$ días frente a $8,78 \pm 12,95$ en HR ( $p=0,005$, IC al 95\% $[0,17 ; 1,65])$, y la Epo también fue inferior en $\mathrm{H} 1$ que en HR: $3,62 \pm 6,14$ vs $4,22 \pm 6,68$ días $(p=0,015)$, evidenciando que - al considerar como variables proxy de la eficiencia tanto la Em como la Epolos hospitales $\mathrm{H} 1$ generaban menos estancias $\mathrm{y}$, por tanto, serían más eficientes (tabla 2). Dado que debería analizarse si la complejidad de los pacientes era similar -mediante la evaluación del NDS y NP- se comprobó que no existía una diferencia claramente significativa $(p=0,055)$ en el NDS $(5,23 \pm 2,88$ vs $5,42 \pm 3,28)$, pero sí en el NP $(3,79 \pm 2,50$ vs $3,55 \pm 2,69)(p=0,002)$, por tanto la comorbilidad de los pacientes de $\mathrm{H} 1$ no era distinta de $\mathrm{HR}$, pero sí había una tendencia clara a realizar más actuaciones diagnósticoterapéuticas (tabla 2).

En la tabla 3 se reflejan los principales indicadores evaluados, según el tipo de hospital y para cada GDR; el análisis por estratos mostrado en dichas tablas arrojó diferencias estadísticamente significativas $(p<0,001)$ en todos los casos y variables, excepto en la mortalidad (no significativa [NS]).

En la tabla 4 se expresan las variables que se encontraron asociadas a ineficiencia, según el modelo de regresión logís-

Tabla 2 Diferencias en estancia y estancia preoperatoria, NDS y NP entre hospitales de nivel 1 y el resto

\begin{tabular}{llll}
\hline & $\mathrm{H} 1$ & $\mathrm{HR}$ & $\mathrm{p}$ \\
\hline Estancia & $7,87 \pm 11,01$ & $8,78 \pm 12,95$ & 0,005 \\
E. Preoperatoria & $3,61 \pm 6,13$ & $4,22 \pm 6,68$ & 0,015 \\
NDS & $5,23 \pm 2,88$ & $5,42 \pm 3,28$ & 0,055 \\
NP & $3,79 \pm 2,50$ & $3,55 \pm 2,69$ & 0,002 \\
\hline
\end{tabular}

H1: nivel I, HR: resto, NDS: número de diagnósticos secundarios, NP: número de procedimientos. Fuente: Instituto de Información Sanitaria, Ministerio de Sanidad y Política Social, 2007. tica binaria construido, observándose que su probabilidad aumentaba 1,38 veces por cada día de Epo que transcurría, en 1,098 veces por cada nuevo diagnóstico, en 1,069 veces por cada nuevo procedimiento, siendo 1,7 veces más probable cuando el acceso era urgente y 1,4 veces cuando se producía en los HR. Globalmente, tuvieron criterio de ineficiencia el $24,1 \%$ de los casos.

En la tabla 5 se muestran los indicadores de los pacientes que fueron dados de alta a su domicilio $(93,4 \%)$ y los fallecidos $(4,5 \%)$, observándose diferencias estadísticamente significativas $(p=0,001)$ siendo menores las Em, Epo, NDS y NP en los primeros.

\section{Discusión}

La clasificación de pacientes mediante GDR se realiza para definir grupos clínicamente comparables, y es una herramienta muy útil para evaluar y medir la calidad de los recursos que se han utilizado en la gestión de un determinado proceso de salud, pudiendo comparar la efectividad y la eficiencia (benchmarking) de un servicio clínico o intervención concreta, conociendo en qué casos el consumo de recursos excede el establecido como norma y, a partir de ahí, introducir los mecanismos correctores correspondientes ${ }^{11}$.

Los GDR están basados en la agrupación de procesos con usos y costes similares, evaluados a través de unas variables proxy (estancia y peso relativo) utilizadas como predictoras del consumo, pero este modelo no recoge la existencia de días de hospitalización innecesarios, que deberían ser identificados en aras de la calidad y la eficiencia. Los estándares de estancia por proceso no detectan el uso inadecuado del episodio, habiendo casos que difieren claramente en el empleo de recursos - alcanzando costes distintos u originando una permanencia más prolongada- por lo que esta variable puede entenderse como proxy de esos costes, al menos de los directos, siendo la duración de la estancia el indicador del CMBD que mejor explica su elevada variabilidad interna ${ }^{12,13}$.

En la construcción del GDR se utilizan el diagnóstico principal, los secundarios y los procedimientos empleados, 
Tabla 3 Principales indicadores evaluados, según tipo de hospital y GDR, España 2007

\begin{tabular}{|c|c|c|c|c|}
\hline GDR & Indicador & $\mathrm{H} 1$ & $\mathrm{HR}$ & $\mathrm{p}$ \\
\hline \multirow[t]{5}{*}{115} & Estancia & $9,69 \pm 9,76$ & $12,04 \pm 8,86$ & 0,001 \\
\hline & E. preoperatoria & $5,21 \pm 6,42$ & $5,25 \pm 7,08$ & NS \\
\hline & NDS & $4,93 \pm 2,54$ & $4,99 \pm 2,83$ & NS \\
\hline & NP & $3,98 \pm 2,31$ & $3,46 \pm 1,86$ & 0,01 \\
\hline & Mortalidad & 10,5 & 10,8 & NS \\
\hline \multirow[t]{5}{*}{116} & Estancia & $5,87 \pm 5,62$ & $6,29 \pm 5,82$ & 0,01 \\
\hline & E. preoperatoria & $2,75 \pm 3,67$ & $3,86 \pm 4,62$ & 0,001 \\
\hline & NDS & $4,93 \pm 2,54$ & $4,98 \pm 2,83$ & NS \\
\hline & NP & $3,98 \pm 2,31$ & $3,46 \pm 1,86$ & 0,001 \\
\hline & Mortalidad & 10,7 & 11,8 & 0,01 \\
\hline \multirow[t]{5}{*}{117} & Estancia & $3,77 \pm 3,97$ & $5,79 \pm 6,50$ & 0,001 \\
\hline & E. preoperatoria & $1,49 \pm 2,65$ & $2,82 \pm 4,48$ & 0,001 \\
\hline & NDS & $4,16 \pm 3,22$ & $4,76 \pm 3,11$ & NS \\
\hline & NP & $2,17 \pm 1,73$ & $2,09 \pm 1,73$ & NS \\
\hline & Mortalidad & 10,3 & 10,7 & NS \\
\hline \multirow[t]{5}{*}{118} & Estancia & $3,19 \pm 3,58$ & $5,09 \pm 5,15$ & 0,001 \\
\hline & E. preoperatoria & $1,53 \pm 2,14$ & $3,26 \pm 3,44$ & 0,01 \\
\hline & NDS & $3,86 \pm 2,51$ & $3,42 \pm 2,50$ & 0,05 \\
\hline & NP & $2,73 \pm 1,84$ & $1,63 \pm 1,21$ & 0,001 \\
\hline & Mortalidad & 10,5 & 10,9 & NS \\
\hline \multirow[t]{5}{*}{549} & Estancia & $22,58 \pm 22,27$ & $24,59 \pm 24,32$ & NS \\
\hline & E. preoperatoria & $7,51 \pm 11,42$ & $10,17 \pm 12,55$ & 0,04 \\
\hline & NDS & $8,33 \pm 2,65$ & $8,94 \pm 3,03$ & 0,01 \\
\hline & NP & $4,48 \pm 3,17$ & $6,09 \pm 4,28$ & 0,001 \\
\hline & Mortalidad & 23,6 & 19,5 & 0,05 \\
\hline
\end{tabular}

E. preoperatoria: estancia preoperatorio, NDS: número de diagnósticos secundarios; NP: número de procedimientos; Mortalidad (por mil); Instituto de Información Sanitaria, Ministerio de Sanidad y Política Social, 2007.

Tabla 4 Variables asociadas a ineficiencia en la implantación de marcapasos, España 2007

\begin{tabular}{|c|c|c|c|c|c|c|}
\hline & B & ET & Wald & gl & Sign. & OR \\
\hline Edad & 0,000 & 0,003 & 0,004 & 1 & 0,951 & 1,000 \\
\hline E. Preop. & 0,321 & 0,008 & 1535,046 & 1 & 0,001 & 1,378 \\
\hline NDS & 0,093 & 0,010 & 91,920 & 1 & 0,001 & 1,098 \\
\hline NP & 0,066 & 0,011 & 34,053 & 1 & 0,001 & 1,069 \\
\hline I. Urgente & 0,578 & 0,067 & 73,970 & 1 & 0,001 & 1,782 \\
\hline $\mathrm{H} 1$ & 0,335 & 0,066 & 25,922 & 1 & 0,001 & 1,398 \\
\hline Constante & $-3,846$ & 0,255 & 228,153 & 1 & 0,001 & 0,021 \\
\hline
\end{tabular}

B: parámetro estimado -implantación ineficiente-; E. preop: estancia preoperatoia; ET: error standard; Wald: método de regresión utilizado; gl: grados de libertad; I. urgente: ingreso urgente, H1: hospitales de nivel I; OR: odds ratio; Sign: significación estadística; NDS: número de diagnósticos secundarios, NP: número de procedimientos; Instituto de Información Sanitaria, Ministerio de Sanidad y Política Social, 2007.

Tabla 5 Diferencias en edad, estancia, estancia preoperatoria, número de diagnósticos y procedimientos, entre supervivientes y fallecidos

\begin{tabular}{lllllll}
\hline & Número & Edad & Estancia & E. Prep & NDS & NP \\
\hline Vivos & 22118 & $75,65 \pm 9,60$ & $8,40 \pm 12,29$ & $4,05 \pm 6,38$ & $5,23 \pm 3,16$ & $3,41 \pm 2,48$ \\
Fallecidos & 1036 & $73,30 \pm 10,12$ & $15,88 \pm 20,36$ & $6,45 \pm 10,04$ & $9,21 \pm 3,13$ & $6,71 \pm 4,30$ \\
$p$ & - & 0,01 & 0.001 & 0,001 & 0,001 & 0,001 \\
\hline
\end{tabular}

E. Preop: estancia preoperatorio, NDS: número de diagnósticos secundarios; NP: número de procedimientos; Instituto de Información Sanitaria, Ministerio de Sanidad y Política Social, 2007. 
midiéndose así las complicaciones (durante la estancia) y las comorbilidades (desde el ingreso), que influyen en su duración, resultados quirúrgicos, presentación de morbilidad sobreañadida, estado funcional final y calidad de vida, reingresos hospitalarios y mortalidad. La complejidad la representa el propio GDR a través del peso relativo y la estancia, las complicaciones son explicadas por el número de NDS y el de NP, y las características socioeconómicas y demográficas se asumen mediante la edad y el género, mientras las del proceso asistencial se evalúan con variables específicas como el tipo de ingreso (urgente o programado) y los reingresos ${ }^{12,13}$. La mayor complejidad de las circunstancias del enfermo se correspondería con un incremento de la Em, de aquí la importancia que se debe conceder a su evaluación y medición en un determinado proceso y, en el caso de los quirúrgicos, tanto la global como la Epo, cuya relación con la primera es evidente ${ }^{14}$.

La asignación de recursos a una determinada actividad implica el coste de oportunidad de no poder utilizarlos en otras, lo que justifica la necesidad de prestar los servicios con efectividad y eficiencia, consumiendo exclusivamente la cantidad mínima de aquellos. En este sentido, se hace imprescindible el empleo de indicadores (bien de proceso, bien de resultado) como herramienta de gestión, lo que conlleva enormes ventajas para gestores y responsables clínicos. Así, el indicador de proceso mide la eficiencia al compararlo con un estándar, en tanto el de resultado mide el impacto sobre la salud del paciente ${ }^{15,16}$.

La ineficiencia técnica se debe, generalmente, a un excesivo empleo de los inputs, en tanto que la asignativa está originada por su uso en proporciones incorrectas. Uno de los indicadores de la primera es el análisis de ratios que, aunque presenta limitaciones importantes especialmente a nivel hospitalario (factores demográficos como el envejecimiento, o geográficos como la ubicación del centro), no impide que se considere un adecuado mecanismo de control ${ }^{15,16}$.

Comprobamos que nuestros resultados, obtenidos a través del $C M B D^{10}$, son concordantes con los reflejados por la $\mathrm{BNDM}^{6}$ : así, la relación entre primoimplantes $(74,61 \%)$ y recambios $(25,39 \%)$ y las edades medias $(76,12$ años para los primeros, 76,96 para los segundos) de este registro son muy similares con nuestro análisis: actividades $(77,81 \%$ implantes, $22,19 \%$ recambios) y edades (76,66 y 77,31 años), respectivamente.

Según la BNDM, el 75,54\% de las intervenciones se efectúa en los grupos etarios de 70-89 años y el 5,17\% en los mayores de 90 , en tanto que nuestros resultados demuestran que en esas dos décadas se hacen el 75,40\% de las actuaciones y solo $3,58 \%$ en los mayores de 90 años $^{6}$. La tasa de MPP de la BNDM es de 680,4 por millón de habitantes y la obtenida en este trabajo de 526,6, pero sin evaluar aquellos implantados en menores de 45 años $^{6}$. En nuestro estudio se observa que los GDR que alcanzan mayor número de complicaciones y comorbilidades $-\mathrm{y}$, por tanto, más elevadas complejidad y mortalidad- son el 115-lógico al tratarse de pacientes con síndrome coronario agudo, insuficiencia cardíaca o shock, acompañados por trastornos de conducción- y el 549, que concentra los MPP complicados y con comorbilidad. El GDR 549 es el que, por configurarse con implantes y revisiones acompañados de complicaciones mayores, muestra indicadores más complejos y tiene el mayor peso relativo $(6,9436)$ y la más elevada mortalidad (268,0 por mil). Ambos GDR (115 y 549) muestran, asimismo, valores de ineficiencia $(30,8 \%$ y $32,7 \%$, respectivamente) superiores a la media $(24,1 \%)$. Los hospitales $\mathrm{H} 1$ tan solo cubren el 3,3\% (115) y 5,7\% (116) de los implantes, y el $8,7 \%$ (117) y 3,9\% (118) de los recambios, expresando la escasa actividad en esta línea de producción, lo que debería hacer reflexionar a gestores y responsables sanitarios. Resulta curioso que en los $\mathrm{H} 1$ se concentra tan solo el 5,3\% de la actividad implantadora de MPP, pero mientras el GDR 117 (control pero sin recambio) llega al 8,7\%, tan solo se colocan el 3,9\% de los nuevos generadores.

Todos los GDR analizados, excepto el 549, presentan Em y Epo significativamente menores en los $\mathrm{H} 1$ que en los $\mathrm{HR}$, con práctica igualdad de NDS. De estos resultados se deduce que el primoimplante en hospitales de nivel I es más eficiente - a pesar de coexistir con similar comorbilidad- pudiendo evitarse así la necesidad de traslado a otro centro sanitario y el consiguiente retraso en la intervención, que podría acarrear complicaciones graves, molestias para el paciente y sus familiares y, por supuesto, mayor gasto para una misma actividad. Los cambios de generador (GDR 117 y 118) ofrecen parámetros parecidos y son, asimismo, más eficientes en estos centros de nivel I.

Debe también comentarse dónde realizar los implantes y las revisiones: según la literatura, las diferencias entre disponer de una estructura quirúrgica o salas de hemodinámica y arritmias, dedicadas multidisciplinarmente a estas actividades, son muy escasas ${ }^{17-19}$. Aunque la bibliografía ofrece la posibilidad de realizar estas intervenciones en programas de cirugía mayor ambulatoria, lo cierto es que no existen disparidades en resultados clínicos ni en morbimortalidad ${ }^{20-24}$.

Existen evidentes limitaciones en este trabajo: en primer lugar, de su propio diseño, al tratarse de un estudio observacional, precisando futuros análisis de corte analítico. Asimismo falta información en el CMBD sobre las posibilidades de infraestructura que ofrecen los hospitales $\mathrm{H} 1$ : existencia de quirófano o sala específica para los implantes, presión quirúrgica, plantillas adecuadas para contemplar la carga laboral (consulta, implantación, postoperatorio). Finalmente, el empleo de los GDR -como herramienta de medición de la actividad- supone trasladar ineficiencias a todas las unidades asistenciales implicadas y no exclusivamente a la responsable del implante.

\section{Conclusiones}

Según nuestro análisis, se deben impulsar estrategias organizativas en los hospitales de nivel I para gestionar los implantes de MPP y los cambios de generador - con su correspondiente seguimiento- realizando ambas técnicas a todos los pacientes que los precisen sin depender del modelo hospitalario sino de la cualificación y preparación técnica de sus profesionales, evitando así retrasos y traslados absolutamente innecesarios cuando se cuenta con recursos especializados y estructuras adecuadas para conseguirlo con seguridad y calidad.

De estos datos se deriva que existe una hipótesis muy interesante para verificar, pero no se puede establecer - al tratarse de un trabajo observacional- una asociación causal entre las variables de eficiencia y los factores estudiados; no obstante, nuestros resultados ratifican que 
existe mayor eficiencia para este proceso en los hospitales más pequeños, con consistencia interna por subgrupos. Este trabajo plantea y deja abierta la hipótesis para comparar, en futuros estudio de corte analítico, los implantes de MPP en cohortes de pacientes con igual comorbilidad entre hospitales de distinto nivel.

\section{Financiación}

El presente trabajo no ha sido financiado por ninguna entidad pública ni privada.

\section{Conflicto de intereses}

Los autores declaran no tener ningún conflicto de intereses.

\section{Bibliografía}

1. Ley $16 / 2003$, de 28 de mayo, de Cohesión y Calidad del Sistema Nacional de Salud. BOE núm. 128, de 29 de mayo de 2003.

2. Osakidetza/Servicio vasco de salud. Manual de descripción de los Grupos Relacionados por el Diagnóstico (AP-GRD v. 18.0). Bilbao: Osakidetza; 2003.

3. Coma Samartín R, Martínez Ferrer J, Sancho-Tello de Carranza MJ, Ruiz Mateas F, Leal del Ojo González J. Registro Español de Marcapasos. VI Informe Oficial de la Sección de Estimulación Cardíaca de la Sociedad Española de Cardiología (2008). Rev Esp Cardiol. 2009;62:1450-63.

4. García Urra F. Registro MAMI: experiencia piloto. Med Intensiva. 1997;21:245-8.

5. Zubía Olaskoaga F, García Urra F. Informe del Registro MAMI (base de datos de marcapasos definitivos en Medicina Intensiva) 1996-2003. Med Intensiva. 2005;29:265-71.

6. Disponible en: www.marcapasossec.orgwww.marcapasossec. org11/01/2010.

7. Real Decreto $643 / 1993$, de 3 de mayo, sobre productos sanitarios implantables activos, BOE de 27 de mayo de 1993.

8. Real Decreto 414/1996, de 1 de marzo, por el que se regulan los productos sanitarios y sus posteriores modificaciones; BOE de 24 de abril de 1996.

9. Grupo de Trabajo de la Sociedad Europea de Cardiología (ESC) sobre marcapasos y terapia de resincronización cardíaca (Desarrollada en colaboración con la European Heart Rhythm Association). Guías europeas de práctica clínica sobre marcapasos y terapia de resincronización cardíaca. Rev Esp Cardiol. 2007;60, 1272 e1-e51.

10. CMBD 2007, Instituto de Información Sanitaria, Ministerio de Sanidad y Política Social, Madrid: 2009.
11. Fetter RB, Shin Y, Freeman JL, Averill RF, Thompson JD. Case mix definition by diagnosis-related groups. Med Care. 1980;18(supl 2):1-53.

12. Peiró S, Meneu R, Roselló ML, Martínez E, Portella E. ¿Qué mide la estancia media de los grupos relacionados por el diagnóstico? Med Clin (Barc). 1994;103:413-7.

13. Cots F, Castell S, García A, Sáez M. Relación de los costes directos de la hospitalización con la duración de la estancia. Gac Sanitaria. 1997;11:287-95.

14. Moreno Millán E. Variación de la estancia preoperatoria en España según grupos de edad, sexo y modo de acceso hospitalario (urgente o programado). Rev Calidad Asistencial. 2008;23:222-9.

15. Martín Martín JJ, López del Amo González MP. La medida de la eficiencia en las organizaciones sanitarias. Presupuesto y Gasto Público. 2007;49:139-61.

16. García Cornejo B. Análisis de la eficiencia del sector hospitalario: una revisión de métodos. Cuadernos Estudios Empresariales. 1997;7:151-76.

17. Yamamura KH, Kloosterman EM, Alba J, García F, Williams PL, Mitran RD, et al. Analysis of charges and complications of permanent pacemaker implantation in the cardiac catheterization laboratory versus the operating room. Pacing Clin Electrophysiol. 1999;22:1820-4.

18. Kiviniemi MS, Pirnes MA, Eranen HJ, Kettunen RV, Hartikainen JE. Complications related to permanent pacemaker therapy. Pacing Clin Electrophysiol. 1999;22:711-20.

19. Zaheri Beryanaki M, Blasco J, Vázquez A, Varela A, Rojas P. Proceso de implantación de marcapasos permanentes en un hospital comarcal. Rev Cal Asistencial. 2008;23: 40-2.

20. García Hernández N. ¿Quién debe implantar el marcapaso definitivo, el cardiólogo o el cirujano cardiovascular? Arch Cardiología México. 2002;72:279-81.

21. Villalba S, Roda J, Quesada A, Palanca V, Zaragoza C, Bataller E, et al. Estudio retrospectivo de pacientes sometidos a implante de marcapasos en cirugía mayor ambulatoria y de corta estancia. Seguimiento a largo plazo y análisis de costes. Rev Esp Cardiol. 2004;57:234-40.

22. Robledo Nolasco R, Méndez Mendoza F, Ruiz Soto JC, Trujillo Cortés $R$, Blanco Canto $M$, Jiménez Valverde $A$, et al. Implantación de marcapasos definitivos en programa de cirugía ambulatoria. Arch Cardiol Mex. 2005;75: 290-5.

23. Mond HG, Irwin M, Ector $\mathrm{H}$, Proclemer A. The world survey of cardiac pacing and cardioverter-defibrillators: calendar year 2005 an International Cardiac Pacing and Electrophysiology Society (ICPES) project. Pacing Clin Electophysiol. 2008;31:1202-12.

24. Hackett D. BCS Working Group on Cardiology Workforce Requirements. British Cardiac Society. 2005. 